\title{
Pre-existing immunity to SARS-CoV-2: the knowns and unknowns
}

Alessandro Sette ${ }^{1,2 凶}$ and Shane Crotty (1) $^{1,2 凶}$

T cell reactivity against SARS-CoV-2 was observed in unexposed people; however, the source and clinical relevance of the reactivity remains unknown. It is speculated that this reflects $T$ cell memory to circulating 'common cold' coronaviruses. It will be important to define specificities of these $T$ cells and assess their association with COVID-19 disease severity and vaccine responses.

As data start to accumulate on the detection and characterization of SARS-CoV-2 T cell responses in humans, a surprising finding has been reported: lymphocytes from $20-50 \%$ of unexposed donors display significant reactivity to SARS-CoV-2 antigen peptide pools ${ }^{1-4}$.

In a study by Grifoni et al. ${ }^{1}$, reactivity was detected in $50 \%$ of donor blood samples obtained in the USA between 2015 and 2018, before SARS-CoV-2 appeared in the human population. T cell reactivity was highest against proteins other than the coronavirus spike protein, but $\mathrm{T}$ cell reactivity was also detected against spike. The SARS-CoV-2 T cell reactivity was mostly associated with $\mathrm{CD} 4^{+} \mathrm{T}$ cells, with a smaller contribution by $\mathrm{CD}^{+} \mathrm{T}$ cells ${ }^{1}$. Similarly, in a study of blood donors in the Netherlands, Weiskopf et al. ${ }^{2}$ detected $\mathrm{CD} 4^{+} \mathrm{T}$ cell reactivity against SARS-CoV-2 spike peptides in 1 of 10 unexposed subjects and against SARS-CoV-2 nonspike peptides in 2 of 10 unexposed subjects. $\mathrm{CD}^{+} \mathrm{T}$ cell reactivity was observed in 1 of 10 unexposed donors. In a third study, from Germany, Braun et al. ${ }^{3}$ reported positive $\mathrm{T}$ cell responses against spike peptides in $34 \%$ of SARS-CoV-2 seronegative healthy donors. Finally, a study of individuals in Singapore, by Le Bert et al. ${ }^{4}$, reported $\mathrm{T}$ cell responses to nucleocapsid protein nsp7 or nsp13 in $50 \%$ of subjects with no history of SARS, COVID-19, or contact with patients with SARS or COVID-19. A study by Meckiff using samples from the UK also detected reactivity in unexposed subjects ${ }^{5}$. Taken together, five studies report evidence of pre-existing $\mathrm{T}$ cells that recognize SARS-CoV-2 in a significant fraction of people from diverse geographical locations.

These early reports demonstrate that substantial $T$ cell reactivity exists in many unexposed people; nevertheless, data have not yet demonstrated the source of the $\mathrm{T}$ cells or whether they are memory $\mathrm{T}$ cells. It has been speculated that the SARS-CoV-2-specific T cells in unexposed individuals might originate from memory T cells derived from exposure to 'common cold' coronaviruses (CCCs), such as $\mathrm{HCoV}-\mathrm{OC} 43, \mathrm{HCoV}-\mathrm{HKU} 1$, HCoV-NL63 and HCoV-229E, which widely circulate in the human population and are responsible for mild self-limiting respiratory symptoms. More than $90 \%$ of the human population is seropositive for at least three of the CCCs ${ }^{6}$. Thiel and colleagues ${ }^{3}$ reported that the $\mathrm{T}$ cell reactivity was highest against a pool of SARS-CoV-2 spike peptides that had homology to CCCs.

What are the implications of these observations? The potential for pre-existing crossreactivity against COVID-19 in a fraction of the human population has led to extensive speculation. Pre-existing T cell immunity to SARS-CoV-2 could be relevant because it could influence COVID-19 disease severity. It is plausible that people with a high level of pre-existing memory $\mathrm{CD}^{+}$ $\mathrm{T}$ cells that recognize SARS-CoV-2 could mount a faster and stronger immune response upon exposure to SARS-CoV-2 and thereby limit disease severity. Memory $\mathrm{T}$ follicular helper $\left(\mathrm{T}_{\mathrm{FH}}\right) \mathrm{CD} 4^{+} \mathrm{T}$ cells could potentially facilitate an increased and more rapid neutralizing antibody response against SARS-CoV-2. Memory $\mathrm{CD}^{+}$and $\mathrm{CD}^{+} \mathrm{T}$ cells might also facilitate direct antiviral immunity in the lungs and nasopharynx early after exposure, in keeping with our understanding of antiviral $\mathrm{CD} 4^{+}$ $\mathrm{T}$ cells in lungs against the related SARS- $\mathrm{CoV}^{7}$ and our general understanding of the value of memory $\mathrm{CD}^{+}$ $\mathrm{T}$ cells in protection from viral infections. Large studies in which pre-existing immunity is measured and correlated with prospective infection and disease severity could address the possible role of pre-existing $\mathrm{T}$ cell memory against SARS-CoV-2.

If the pre-existing $\mathrm{T}$ cell immunity is related to CCC exposure, it will become important to better understand the patterns of CCC exposure in space and time. It is well established that the four main CCCs are cyclical in their prevalence, following multiyear cycles, which can differ across geographical locations ${ }^{8}$. This leads to the speculative hypothesis that differences in CCC geo-distribution might correlate with burden of COVID-19 disease severity. Furthermore, highly speculative hypotheses related to pre-existing memory $\mathrm{T}$ cells can be proposed regarding COVID-19 and age. Children are less susceptible to COVID-19 clinical symptoms. Older people are much more susceptible to fatal COVID-19. The reasons for 
both are unclear. The age distribution of CCC infections is not well established and CCC immunity should be examined in greater detail. These considerations underline how multiple variables may be involved in potential pre-existing partial immunity to COVID-19 and multiple hypotheses are worthy of further exploration, but caution should be exercised to avoid overgeneralizations or conclusions in the absence of data.

Pre-existing $\mathrm{CD}^{+} \mathrm{T}$ cell memory could also influence vaccination outcomes, leading to a faster or better immune response, particularly the development of neutralizing antibodies, which generally depend on $\mathrm{T}$ cell help. At the same time, pre-existing $\mathrm{T}$ cell memory could also act as a confounding factor, especially in relatively small phase I vaccine trials. For example, if subjects with pre-existing reactivity were assorted unevenly in different vaccine dose groups, this might lead to erroneous conclusions. Obviously, this could be avoided by considering pre-existing immunity as a variable to be considered in trial design. Thus, we recommend measuring pre-existing immunity in all COVID-19 vaccine phase I clinical trials. Of note, such experiments would also offer an exciting opportunity to ascertain the potential biological significance of pre-existing SARS-CoV-2-reactive T cells.

It is frequently assumed that pre-existing $\mathrm{T}$ cell memory against SARS-CoV-2 might be either beneficial or irrelevant. However, there is also the possibility that pre-existing immunity might actually be detrimental, through mechanisms such as 'original antigenic sin' (the propensity to elicit potentially inferior immune responses owing to pre-existing immune memory to a related pathogen), or through antibody-mediated disease enhancement. While there is no direct evidence to support these outcomes, they must be considered. A detrimental effect linked to pre-existing immunity is eminently testable and would be revealed by the same COVID-19 cohort and vaccine studies proposed above.

There is substantial data from the influenza literature indicating that pre-existing cross-reactive $\mathrm{T}$ cell immunity can be beneficial. In the case of the H1N1 influenza pandemic of 2009, it was noted that an unusual ' $V$ '-shaped age distribution curve existed for disease severity, with older people faring better than younger adults. This correlated with the circulation of a different
H1N1 strain in the human population decades earlier, which presumably generated pre-existing immunity in people old enough to have been exposed to it. This was verified by showing that pre-existing immunity against H1N1 existed in the general human population ${ }^{9,10}$. It should be noted that if some degree of pre-existing immunity against SARS-CoV-2 exists in the general population, this could also influence epidemiological modelling, and suggests that a sliding scale model of COVID-19 susceptibility may be considered.

In conclusion, it is now established that SARS-CoV-2 pre-existing immune reactivity exists to some degree in the general population. It is hypothesized, but not yet proven, that this might be due to immunity to CCCs. This might have implications for COVID-19 disease severity, herd immunity and vaccine development, which still await to be addressed with actual data.

1. Grifoni, A. et al. Targets of T cell responses to SARS-CoV-2 coronavirus in humans with COVID-19 disease and unexposed individuals. Cell 181, 1489-1501 (2020).

2. Weiskopf, D. et al. Phenotype of SARS-CoV-2-specific T-cells in COVID-19 patients with acute respiratory distress syndrome. Preprint at medRxiv https://doi.org/10.1101/2020.04.11 20062349 (2020).

3. Braun, J. et al. Presence of SARS-CoV-2 reactive T cells in COVID-19 patients and healthy donors. Preprint at medRxiv https://doi.org/ $10.1101 / 2020.04 .17 .20061440$ (2020).

4. Le Bert, N. et al. Different pattern of pre-existing SARS-COV-2 specific $\mathrm{T}$ cell immunity in SARS-recovered and uninfected individuals. Preprint at bioRxiv https://doi.org/10.1101/2020. 05.26.115832 (2020).

5. Meckiff, B. J. et al. Single-cell transcriptomic analysis of SARS-CoV-2 reactive $\mathrm{CD}^{+}{ }^{+} \mathrm{T}$ cells. Preprint at bioRxiv https://doi.org/10.1101/ 2020.06.12.148916 (2020).

6. Gorse, G. J., Patel, G. B., Vitale, J. N. \& O'Connor, T. Z. Prevalence of antibodies to four human coronaviruses is lower in nasal secretions than in serum. Clin Vaccine Immunol 17, 1875-1880 (2010).

7. Zhao, J. et al. Airway memory CD4(+) T cells mediate protective immunity against emerging respiratory coronaviruses. Immunity $\mathbf{4 4}$ 1379-1391 (2016).

8. Killerby, M. E. et al. Human coronavirus circulation in the United States 2014-2017. J. Clin. Virol. 101, 52-56 (2018).

9. Greenbaum, J. A. et al. Pre-existing immunity against swine-origin H1N1 influenza viruses in the general human population. Proc. Natl Acad. Sci. USA 106, 20365-20370 (2009).

10. Hancock, K. et al. Cross-reactive antibody responses to the 2009 pandemic H1N1 influenza virus. N. Engl. J. Med. 361, 1945-1952 (2009).

\section{Acknowledgements}

This work was funded by the NIH NIAID under awards U 19 Al42742 (Cooperative Centers for Human Immunology) and contract no. 75N9301900065.

\section{Competing interests}

A.S. is a consultant for Gritstone, Flow Pharma and Avalia. S.C. declares no competing interests. 